\title{
The Generalized Plasma in One Dimension: Evaluation of a Partition Function
}

\author{
D. M. Bressoud ${ }^{1, \star}$ and I. P. Goulden ${ }^{2}$ \\ ${ }^{1}$ Department of Mathematics, The Pennsylvania State University, University Park, \\ PA 16802, USA \\ ${ }^{2}$ Department of Mathematics, University of Waterloo, Waterloo, Ontario N2L 3G1, Canada
}

\begin{abstract}
Forrester and Jancovici have given sum rules for a two-dimensional generalized plasma with two species of particles interacting through logarithmic potentials with three independent coupling constants. They have also found a specific one-dimensional solvable model which satisfies the analogs of their sum rules. A class of one-dimensional models for which the partition function is evaluable is given as well as a more general result evaluating multidimensional integrals.
\end{abstract}

\section{Introduction}

Forrester and Jancovici [1] have given an exactly solved model for a onedimensional generalized plasma with two species of particles (roman and greek), interacting through logarithmic potentials, with three independent coupling constants. This was motivated by their discovery of sum rules for such a generalized plasma in two dimensions (see also Halperin [2] and Girvin [3]) and the desire to at least verify the one-dimensional analogs of these rules.

The two-dimensional system with roman and greek particles of density $\varrho_{R}$ and $\varrho_{G}$, respectively, and independent coupling constants $g_{R R}, g_{R G}, g_{G G}$ has Hamiltonian

$$
\begin{aligned}
H= & -g_{R R} \sum_{i>j} \ln r_{i j}-g_{G G} \sum_{\alpha>\beta} \ln r_{\alpha \beta}-g_{R G} \sum_{i, \alpha} \ln r_{i \alpha} \\
& +\left(g_{R R} \varrho_{R}+g_{R G} \varrho_{G}\right) \sum_{i} \int \ln \left|\mathbb{R}_{i}-\mathbb{R}\right| d \mathbb{R} \\
& +\left(g_{G G} \varrho_{G}+g_{R G} \varrho_{R}\right) \sum_{\alpha} \int \ln \left|\mathbb{R}_{\alpha}-\mathbb{R}\right| d r \\
& -\left(\frac{1}{2} g_{R R} \varrho_{R}^{2}+\frac{1}{2} g_{G G} \varrho_{G}^{2}+g_{R G} \varrho_{R} \varrho_{G}\right) \int \ln \left|\mathbb{R}-\mathbb{R}^{\prime}\right| d \mathbb{R} d \mathbb{R}^{\prime},
\end{aligned}
$$

where the particle-background and background-background interactions have been chosen in a way which compensates the remote particle-particle interactions

$\star$ Partially supported by N.S.F. grant 
so that we can expect the system to have a well-behaved thermodynamic limit. Forrester and Jancovici's exactly solved model is for the system where all particles lie on a circle of radius $R$ :

$$
z_{j}=\operatorname{Re}^{i \theta_{j}}, \quad z_{\beta}=\operatorname{Re}^{i \theta_{\beta}},
$$

and $g_{R R}=g_{R G}=2, g_{G G}=4$. We shall consider the more general case of this onedimensional model,

$$
g_{R R}=g_{R G}=2 y, \quad g_{G G}=2 y+2, \quad y \in \mathbb{N} .
$$

The excess partition function is

$$
\begin{aligned}
Z= & (2 \pi)^{-(a+b) N} R^{-(y a+(y+1) b) N} \prod_{j=1}^{a N} \int_{0}^{2 \pi} d \theta_{j} \prod_{\alpha=1}^{b N} \int_{0}^{2 \pi} d \theta_{\alpha} \\
& \times \prod_{j<k}\left|e^{i \theta_{j}}-e^{i \theta_{k}}\right|^{2 y} \prod_{\alpha<\beta}\left|e^{i \theta_{\alpha}}-e^{i \theta_{\beta}}\right|^{2 y+2} \prod_{j, \alpha}\left|e^{i \theta_{j}}-e^{i \theta_{\alpha}}\right|^{2 y} .
\end{aligned}
$$

We shall show that

$$
Z=\frac{(a y N+b(y+1) N) ! b N ! R^{-(y a+(y+1) b) N}}{y !^{a N}(y+1) !^{b N}\left(\frac{a y N}{y+1}+1\right)_{b N}}
$$

where $(y)_{n}=y(y+1) \ldots(y+n-1)$.

\section{A General Identity}

Since $\left|e^{i \theta_{j}}-e^{i \theta_{k}}\right|^{2}=\left(1-e^{i\left(\theta_{j}-\theta_{k}\right)}\right)\left(1-e^{i\left(\theta_{k}-\theta_{J}\right)}\right)$, the partition function $Z$ as defined in Eq. (1.2) is simply the power of $R$ times the constant term in

$$
\prod_{j<k}\left(1-\frac{x_{j}}{x_{k}}\right)^{y}\left(1-\frac{x_{k}}{x_{j}}\right)^{y} \prod_{\alpha<\beta}\left(1-\frac{x_{\alpha}}{x_{\beta}}\right)^{y+1}\left(1-\frac{x_{\beta}}{x_{\alpha}}\right)^{y+1} \prod_{j, \alpha}\left(1-\frac{x_{j}}{x_{\alpha}}\right)^{y}\left(1-\frac{x_{a}}{x_{j}}\right)^{y} .
$$

Equation (1.3) follows from a proposition proved in a more general setting by the authors [4].

Proposition 2.1. Let $a_{1}, \ldots, a_{n}$ be positive integers, $A$ be an arbitrary subset of $\{(i, j): 1 \leqq i<j \leqq n\}, \Im_{A}$ be the set of permutations on $\{1, \ldots, n\}$ whose inversions are contained in $A$ :

$$
\mathfrak{S}_{A}=\left\{\sigma \in \mathfrak{S}_{n}: i<j \text { and } \sigma^{-1}(i)>\sigma^{-1}(j) \text { implies }(i, j) \in A\right\},
$$

and let $\chi(T)$ be the characteristic function which is 1 if $T$ is true, 0 otherwise. If [1] denotes the constant term in the succeeding expression, then

$$
\begin{aligned}
\text { [1] } & \prod_{1 \leqq i<j \leqq n}\left(1-\frac{x_{i}}{x_{j}}\right)^{a_{i}}\left(1-\frac{x_{j}}{x_{i}}\right)^{a_{j}-\chi((i, j) \notin A)} \\
& =\frac{\left(a_{1}+a_{2}+\ldots+a_{n}\right) !}{\left(a_{1}-1\right) !\left(a_{2}-1\right) ! \ldots\left(a_{n}-1\right) !} \sum_{\sigma \in \mathfrak{S}_{A}} \prod_{l=1}^{n} \frac{1}{\left(a_{\sigma(1)}+\ldots+a_{\sigma(l)}\right)} .
\end{aligned}
$$


Equation (1.3) is the special case of this proposition where $n=a N+b N$, $A=\{(i, j): \quad 1 \leqq i<j \leqq a N$ or $a N+1 \leqq i<j \leqq a N+b N\}, \quad \Im_{A} \simeq \Im_{a N} \times \Im_{b N}$, and $a_{1}=\ldots=a_{a N}=y, a_{a N+1}=\ldots=a_{a N}+{ }_{b N}=y+1$.

For $A=\{(i, j): 1 \leqq i<j \leqq n\}$, one observes that

$$
\sum_{\sigma \in \mathbb{E}_{n}} \prod_{l=1}^{n} \frac{1}{\left(a_{\sigma(1)}+\ldots+a_{\sigma(l)}\right)}=\frac{1}{a_{1} a_{2} \cdot \ldots \cdot a_{n}} .
$$

This can be proved by induction on $n$. For the inductive step, we observe that the only term involving $\sigma(n)$ is

$$
\left(a_{\sigma(1)}+\ldots+a_{\sigma(n)}\right)^{-1}=\left(a_{1}+\ldots+a_{n}\right)^{-1},
$$

which can be factored out of the summation. The sum is now rewritten as a sum over possible images of $n$ of the sum over all permutations of the remaining $n-1$ elements.

Proposition 2.1 thus implies an identity conjectured by Dyson [5] and proved by Gunson [6] and Wilson [7]:

$$
\text { [1] } \prod_{1 \leqq i<j \leqq n}\left(1-\frac{x_{i}}{x_{j}}\right)^{a_{i}}\left(1-\frac{x_{j}}{x_{i}}\right)^{a_{j}}=\frac{\left(a_{1}+a_{2}+\ldots+a_{n}\right) !}{a_{1} ! a_{2} ! \ldots a_{n} !} .
$$

In fact, the proof of Proposition 2.1 will essentially follow Good's proof [8] of Eq. (2.3).

\section{Proof}

To prove this proposition, we shall need a lemma. Let $T$ be a tournament (a complete directed graph) on $n$ vertices,

$$
T \leqq\{(i, j): 1 \leqq i \neq j \leqq n\}, \quad(i, j) \in T \Leftrightarrow(j, i) \notin T .
$$

We say $T$ is transitive if it contains no cycles and thus corresponds to a permutation, $\sigma$, of $\{1, \ldots, n\}$, where $\sigma(i)$ is the vertex with in-degree $i-1$.

Lemma 3.1. Let $a=\left(a_{1}, \ldots, a_{n}\right), T$ be a tournament on $n$ vertices, then

$$
\begin{aligned}
& \text { [1] } \prod_{(i, j) \in T}\left(1-\frac{x_{i}}{x_{j}}\right)^{a_{i}}\left(1-\frac{x_{j}}{x_{i}}\right)^{a_{j}-1} \\
& = \begin{cases}\frac{\left(a_{1}+\ldots+a_{n}\right) !}{\left(a_{1}-1\right) ! \ldots\left(a_{n}-1\right) !} \prod_{i=1}^{n} \frac{1}{a_{\sigma(1)}+\ldots+a_{\sigma(i)}}, & \text { if } T \text { is transitive, } \\
0, & \text { otherwise. }\end{cases}
\end{aligned}
$$

Proof of Lemma. By the Lagrange interpolation formula (see Good [8]) we have that

$$
1=\sum_{i=1}^{n} \prod_{\substack{j=1 \\ j \neq i}}^{n} \frac{1}{1-\frac{x_{i}}{x_{j}}}
$$


Let $C(T, a)=[1] \prod_{(i, j) \in T}\left(1-\frac{x_{i}}{x_{j}}\right)^{a_{i}}\left(1-\frac{x_{j}}{x_{i}}\right)^{a_{j}-1}$. Multiplying both sides of Eq. (3.2) by $\prod_{(i, j) \in T}\left(1-\frac{x_{i}}{x_{j}}\right)^{a_{i}}\left(1-\frac{x_{j}}{x_{i}}\right)^{a_{j}-1}$ and taking constant terms yields

$$
C(T, a)=\sum_{i=1}^{n} C\left(T, a-\delta_{i}\right)
$$

where $\delta_{i}$ is the unit vector in the $i^{\text {th }}$ direction. We also have the initial conditions that if $a_{k}=0$, then

$$
C(T, a)= \begin{cases}1, & \text { if } k=n=1, \\ C\left(T \backslash k, a_{1}, \ldots, a_{k-1}, a_{k+1}, \ldots, a_{n}\right), & \text { if vertex } k \text { has in-degree } 0,(3.4) \\ 0, & \text { otherwise, }\end{cases}
$$

where $T \backslash k$ means the tournament obtained by removing vertex $k$ and all incident edges. It is readily verified that the right-hand side of Eq. (3.1) also satisfies this recurrence (3.3) and set of initial conditions (3.4).

Proof of Proposition. We begin the proof of Eq. (2.1) by observing that

$$
\begin{aligned}
& \prod_{i<j}\left(1-\frac{x_{i}}{x_{j}}\right)^{a_{i}}\left(1-\frac{x_{j}}{x_{i}}\right)^{a_{j}-\chi((i, j) \notin A)} \\
& =\prod_{i<j}\left(1-\frac{x_{i}}{x_{j}}\right)^{a_{i}}\left(1-\frac{x_{j}}{x_{i}}\right)^{a_{j}-1} \prod_{(i, j) \in A}\left(1-\frac{x_{j}}{x_{i}}\right) .
\end{aligned}
$$

We now consider the formal expansion of

$$
\prod_{(i, j) \in A}\left(1-\frac{x_{j}}{x_{i}}\right)
$$

For each pair $(i, j) \in A$, choosing the first term, 1, will leave the product to the left unchanged. Choosing the second term, $-x_{j} / x_{i}$, yields

$$
\left(1-\frac{x_{i}}{x_{j}}\right)^{a_{i}}\left(1-\frac{x_{j}}{x_{i}}\right)^{a_{j}-1}\left(-\frac{x_{j}}{x_{i}}\right)=\left(1-\frac{x_{j}}{x_{i}}\right)^{a_{j}}\left(1-\frac{x_{i}}{x_{j}}\right)^{a_{i}-1} \text {. }
$$

Thus, choosing the second term has the affect of reversing the order of $i$ and $j$ in the corresponding term of the product. Thus we get that

$$
\prod_{i<j}\left(1-\frac{x_{i}}{x_{j}}\right)^{a_{i}}\left(1-\frac{x_{j}}{x_{i}}\right)^{\left.a_{j}-\chi(i, j) \notin A\right)}=\sum_{T} \prod_{(i, j) \in T}\left(1-\frac{x_{i}}{x_{j}}\right)^{a_{i}}\left(1-\frac{x_{j}}{x_{i}}\right)^{a_{j}-1}
$$

where the sum is over all tournaments on $n$ vertices such that if $(i, j) \in T$ and $j<i$ then $(j, i) \in A$. The proposition follows by taking the constant term of each side of Eq. (3.7) and using Lemma 3.1.

\section{References}

1. Forrester, P.J., Jancovici, B.: Generalized plasmas and the anomalous quantum Hall effect. J. Physique Lett. 45, L-583 (1984)

2. Halperin, B.I.: Theory of quantized Hall conductance. Helv. Phys. Acta 56, 75 (1983)

3. Girvin, S.M.: Nat. Bur. Stand. (preprint) 
4. Bressoud, D.M., Goulden, I.P.: Trans. Am. Math. Soc. 291, 203 (1985)

5. Dyson, F.J.: Statistical theory of the energy levels of complex systems. I. J. Math. Phys. 3, 140 (1962)

6. Gunson, J.: Proof of a conjecture by Dyson in the statistical theory of energy levels. J. Math. Phys. 3, 752 (1962)

7. Wilson, K.: Proof of a conjecture by Dyson. J. Math. Phys. 3, 1040 (1962)

8. Good, I.J.: Short proof of a conjecture by Dyson. J. Math. Phys. 11, 1884 (1970)

Communicated by H. Araki

Received September 30, 1986; in revised form December 17, 1986 
